\section{Vers une thérapie génique pour la cardiomyopathie associée à l'ataxie de Friedreich}

\footnotetext{
${ }^{1}$ Département de médecine translationnelle et neurogénétique, Institut de génétique et de biologie moléculaire et cellulaire (IGBMC), 1, rue Laurent Fries, BP 10142, 67404 Illkirch, France; ${ }^{2}$ Inserm, U596, 1, rue Laurent Fries, BP 10142, 67404 IIIkirch, France ;

${ }^{3}$ CNRS, UMR7104, 1, rue Laurent Fries, BP 10142, 67404 IIIkirch, France;

${ }^{4}$ Université de Strasbourg, Strasbourg, France ;

${ }^{5}$ Collège de France, chaire de génétique humaine, IIIkirch, France; hpuccio@igbmc.fr
}

\section{L'ataxie de Friedreich}

Les ataxies cérébelleuses sont caractérisées par des troubles de la coordination des mouvements volontaires, de l'équilibre et de la mobilité, liés à une atteinte du cervelet et/ou des voies spinocérébelleuses. L'ataxie de Friedreich (AF), la plus fréquente des ataxies héréditaires avec une incidence de 1 : 30 000, est une maladie neurodégénérative progressive incurable [1]. Les signes cliniques sont dominés par l'ataxie, due à la perte de coordination motrice (syndrome cérébelleux) et aux troubles de l'équilibre (perte de la sensibilité profonde), qui évolue jusqu'à la perte de la capacité ambulatoire en moyenne après une dizaine d'années [1]. Ces signes neurologiques sont fréquemment associés à une cardiomyopathie hypertrophique ( $80 \%$ des cas), cause de mortalité précoce pour $60 \%$ des patients [2]. L'ataxie de Friedreich est due à un déficit en frataxine (FXN), une protéine mitochondriale ubiquitaire essentielle à la vie [3]. La mutation principale est une répétition du triplet GAA dans le premier intron du gène $F X N$. Elle induit une répression transcriptionnelle par la formation d'hétérochromatine, qui résulte en une diminution drastique du niveau de FXN, entre 5 et $30 \%$ du niveau normal dans tous les tissus [3]. La FXN joue un rôle essentiel dans la régulation de la synthèse des centres fer-soufre (Fe-S), cofacteurs essentiels à de nombreuses voies cellulaires, notamment la respiration mitochondriale et la régulation du métabolisme du fer. L'étude du déficit en FXN dans différents modèles (levure, cellulaires et murins) a permis de comprendre la physiopathologie de la maladie et de valider des modèles murins d'étude préclinique [4]. Les conséquences physiopathologiques du déficit en FXN sont un déficit en centres Fe-S, un dysfonctionnement mitochondrial global avec diminution de la production d'ATP et accumulation de fer mitochondrial, une dérégulation de l'homéostasie du fer cellulaire, ainsi qu'un stress cellulaire (Figure 1).

À l'heure actuelle, aucun traitement n'est disponible pour les patients atteints d'ataxie de Friedreich, et les stratégies thérapeutiques développées ciblent principalement les conséquences secondaires du déficit en FXN en utilisant des chélateurs du fer ou des anti-oxydants; plus récemment, certaines approches visent à augmenter la quantité de FXN dans les cellules en agissant sur les marques épigénétiques pour ouvrir la chromatine, ou en stabilisant la protéine [5]. Une troisième approche, la thérapie génique, consiste à délivrer dans les cellules d'intérêt une séquence d'acide nucléotidique thérapeutique au moyen d'un vecteur viral modifié. Cette approche est particulièrement prometteuse pour les maladies autosomiques récessives à évolution postnatale lente telles que l'ataxie de Friedreich. En effet, tous les patients ont un taux cellulaire résiduel de FXN entre 5 et $30 \%$ du niveau normal - et une augmentation modérée de celui-ci devrait être thérapeutique. En effet, les porteurs sains hétérozygotes de la mutation n'expriment qu'environ $50 \%$ du niveau normal de FXN. De plus, ce taux résiduel de FXN chez les patients laisse supposer une faible probabilité de réponse immunitaire contre la protéine exogène thérapeutique, ce qui est un élément important à prendre en compte dans la conception d'un essai clinique. Les vecteurs viraux recombinants dérivés des virus adéno-associés (AAV) sont des outils de choix pour le transfert d'un gène thérapeutique in vivo: ce sont des virus non enveloppés à ADN simple brin de la famille des parvoviridae, qui infectent aussi bien les cellules mitotiques que post-mitotiques comme les cardiomyocytes et les neurones. Les AAV ne sont pas pathogènes pour l'homme et seul un très faible pourcentage d'entre eux s'intégreront dans le génome nucléaire [6]. De nombreux sérotypes d'AAV ont été décrits, mais I'AAVrh10 notamment permet un transfert de gène thérapeutique efficace dans les cellules cardiaques et neuronales des ganglions dorsaux rachidiens, les deux principales cibles d'intérêt dans l'ataxie de Friedreich.

\section{Preuve de concept chez l'animal} de l'efficacité d'une thérapie génique dans l'ataxie de Friedreich

Au cours de notre étude [7], nous avons développé une approche de thérapie génique pour le traitement de la cardiomyopathie hypertrophique associée à l'ataxie de Friedreich. Le modèle était celui des souris MCK, chez lesquelles l'inactivation conditionnelle du gène de la frataxine reproduit l'essentiel des caractéristiques de la pathologie cardiaque des patients, bien que plus sévère, ainsi que les caractéristiques physiopathologiques et biochimiques de 


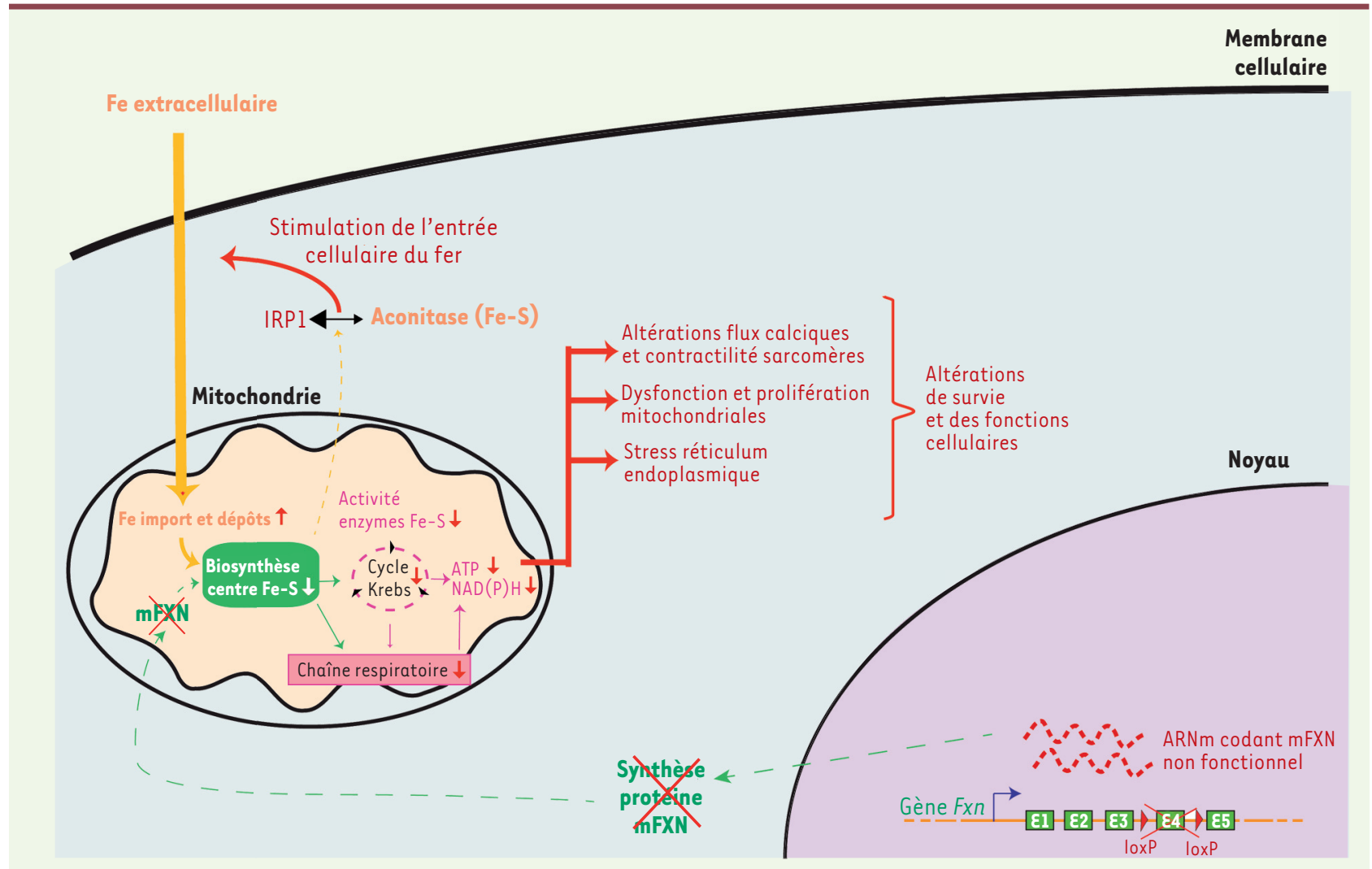

Figure 1. Conséquences cellulaires cardiaques du déficit en FXN. Le déficit induit des dysfonctions biochimiques, métaboliques et fonctionnelles responsables de la perte de fonction et de survie des cellules cardiaques dans le modèle murin conditionel cardiaque. L'inactivation du gène FXN est induite par la délétion conditionnelle basée sur le système Cre-LoxP de l'exon 4, spécifiquement dans les cellules musculaires striées (la recombinase est sous le contrôle du promoteur du gène codant pour la créatine kinase musculaire [MCK]). Le niveau cellulaire en FXN murine $(\mathrm{mFXN})$ est nul dans les cellules exprimant la recombinase, modélisant la maladie sous une forme plus sévère que chez les patients atteints d'ataxie de Friedreich, qui conservent un niveau résiduel en FXN. Le déficit en FXN induit un déficit dans la biosynthèse des centres fer-soufre (Fe$S$ ), et donc de toutes les enzymes dépendant de cette voie. Ceci entraîne une diminution de la chaîne respiratoire et du cycle de Krebs, et donc une réduction de la production d'ATP et de $\operatorname{NAD}(\mathrm{P}) \mathrm{H}$. Ce déficit bioénergétique a de multiples conséquences dont (1) la dysfonction et la prolifération des mitochondries par compensation, (2) l'altération de l'activité contractile des cardiomyocytes, que ce soit au niveau des sarcomères ou du cycle du calcium, (3) l'induction d'un fort stress cellulaire. En outre, le déficit en centres Fe-S induit le basculement de l'aconitase cytosolique, qui est une enzyme à centre $\mathrm{Fe}-\mathrm{S}$, vers sa forme IRPI (iron responsive protein 1). IRPI, par une régulation traductionnelle, induit l'entrée cellulaire du fer $(\mathrm{Fe})$, qui est alors importé dans les mitochondries où son accumulation provoque des dépôts de fer. Ces différents phénomènes pathologiques aboutissent à terme à une dysfonction globale puis à la mort cellulaire.

la pathologie humaine [8] (Figure 1). Ces souris sont asymptomatiques à trois semaines de vie, et développent une dysfonction systolique et une hypertrophie suivie d'une dilatation du ventricule gauche dès cinq semaines. Cette cardiomyopathie évolue en une insuffisance cardiaque vers sept semaines, entraînant un décès prématuré avant 12 semaines (une souris vie normalement 2 à 3 ans).

Dans un premier temps, nous avons traité une cohorte de souris MCK âgées de trois semaines par une injection intraveineuse de $5 \times 10^{13}$ vecteurs génomes/kg du vecteur thérapeutique AAVrh10.CAG-hFXN, exprimant la frataxine humaine ( $h F X N)$ sous le contrôle d'un promoteur fort et ubiquitaire. Ce traitement, pendant la période précédant les symptômes, a permis non seulement de prévenir le développement de la cardiomyopathie, mais aussi celui de toutes les anomalies cellulaires et biochimiques jusqu'à 35 semaines d'âge (date du sacrifice de la cohorte pour analyses histologiques, biochimiques et moléculaires) et au-delà de un an (pour un petit nombre de souris) (Figure 2).

De façon spectaculaire, le traitement des souris MCK âgées de sept semaines, donc déjà attteintes d'insuffisance cardiaque avancée, a permis une correction progressive de la fonction contractile, du débit cardiaque et du remodelage $d u$ ventricule gauche entre sept et 14 semaines jusqu'à leur normalisation, puis leur stabilisation complète jusqu'à 22 semaines. La nette amélioration de la 


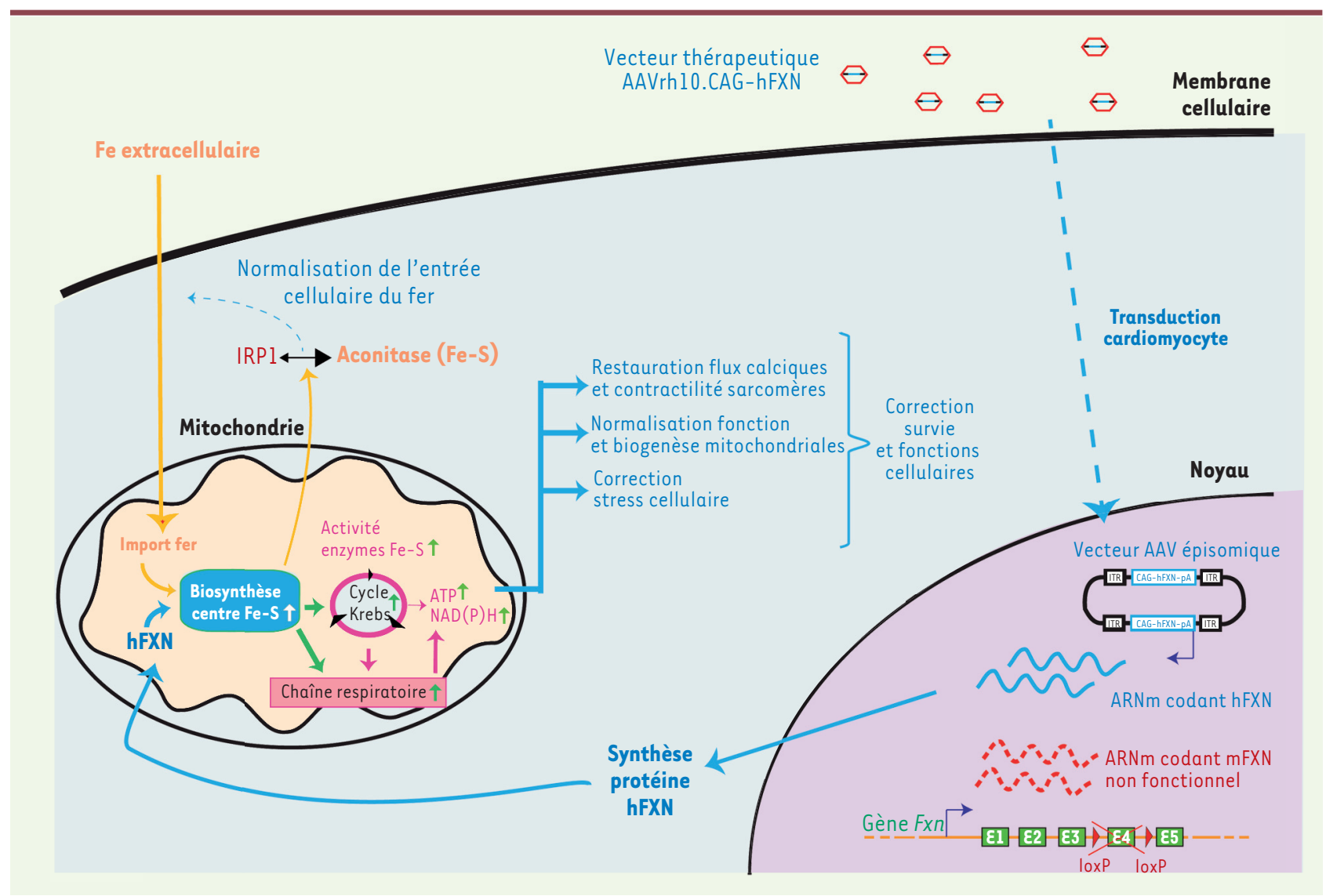

Figure 2. Conséquences de l'administration du vecteur AAVrh10.CAG-hFXN in vivo chez la souris MCK. Le vecteur permet d'apporter une copie fonctionnelle du gène $h F X N$ dans les cellules cardiaques affectées et de corriger les altérations biochimiques, bioénergétiques et fonctionnelles associées au déficit en mFXN. Le vecteur viral recombinant AAVrh10.CAG-hFXN est constitué de la caspide virale du sérotype AAVrh10 et porte une cassette d'expression thérapeutique codant pour la FXN humaine (hFXN) sous le contrôle du promoteur fort et ubiquitaire CAG. Après administration intraveineuse, ce vecteur est capable de transduire efficacement les cardiomyocytes des souris MCK, c'est-à-dire d'entrer dans la cellule où I'ADN thérapeutique sera libéré dans le noyau pour y être transformé en ADN double brin épisomique et concatemérisé, permettant ainsi son expression. La hFXN thérapeutique ainsi produite induit la restauration de la biosynthèse des centres Fe-S et la correction de l'activité des enzymes à centre Fe-S. Ceci permet, d'une part, la restauration de la respiration mitochondriale et, par extension, la normalisation de la fonction et de la biogenèse mitochondriales, et, d'autre part, l'activation de l'aconitase cytosolique et la normalisation du métabolisme du fer (Fe). La correction du statut bioénergétique des cellules cardiaques permet une normalisation progressive de la fonction et de la survie cellulaires.

fonction cardiaque, évaluée par échocardiographie, seulement une semaine après l'administration du vecteur, a été corrélée à une expression substantielle de hFXN, la correction partielle de l'ultrastructure des sarcomères et des mitochondries, ainsi que l'augmentation, puis la correction, de l'activité des enzymes à centre Fe-S. De même, les biomarqueurs relatifs à l'hypertrophie cardiaque (les myosines $\alpha$ et $\beta$, l'actine $\alpha$, et des facteurs de transcription cardiaques tels que GATA4), la signalisation neuro-hormonale (ANP [atrial natriuretic peptide], BNP [brain natriuretic peptide]), la fibrose (collagènes de type 1 et 3 ) et au cycle du calcium (SERCA2A [sarco/endoplasmic reticulum $\mathrm{Ca}^{2+}$ ATPase]), ont tous été normalisés progressivement dans les semaines suivant le traitement (Figure 2). Aucun phénomène de toxicité cardiaque ou périphérique associé au traitement et à la surexpression de la hFXN n'a été mis en évidence. Ces résultats démontrent pour la première fois une correction complète du phénotype cardiaque et biochimique dans un modèle murin d'ataxie de Friedreich par une approche de thérapie génique.

\section{Perspectives dans l'ataxie} de Friedreich humaine

Notre étude établit la preuve de concept de la pertinence et de l'efficacité de la thérapie génique in vivo pour le traitement de la cardiomyopathie associée à l'ataxie de Friedreich, non seulement à visée préventive, mais surtout curative. De plus, nos résultats indiquent que les cellules déficientes en FXN peuvent être corrigées même à un stade très avancé où dominent les altérations biochimiques, structurales et fonctionnelles, démontrant une plasticité importante des cellules 
cardiaques. Nous supposons que cette efficacité thérapeutique repose à la fois sur la rapidité d'expression de la hFXN thérapeutique induite par le vecteur AAVrh10 ainsi que sur la nature hautement dynamique des mitochondries, dont le turnover est très rapide, permettant une sélection rapide des mitochondries fonctionnellement corrigées [9]. Cette possibilité de correction des cellules cardiaques est particulièrement importante dans un contexte clinique, puisque les patients ne seront pas traités en phase pré-symptomatique dans les premiers essais. Afin de transposer cette approche en clinique, plusieurs études sont en cours (1) pour déterminer l'efficacité du vecteur AAVrh10. CAG-hFXN en fonction de sa concentration (effet dose-réponse), et (2) pour optimiser l'efficacité et l'innocuité du mode d'administration chez le gros animal plus proche de la physiologie humaine. Nous continuons nos efforts de développement dans le laboratoire afin d'évaluer une approche similaire sur les symptômes neurologiques. $\diamond$

Toward gene therapy for Friedreich ataxia-associated cardiomyopathy

\section{LIENS D'INTÉR̂ิT}

H. Puccio est l'un des fondateurs scientifiques de AAVLife, une SAS dédiée à la thérapie génique pour les maladies rares.

B. Belbellaa déclare n'avoir aucun lien d'intérêt concernant les données publiées dans cet article.

\section{RÉFÉRENCES}

1. Parkinson MH, Boesch S, Nachbauer W, et al. Clinical features of Friedreich's ataxia: classical and atypical phenotypes. J Neurochem 2013; 126 (suppl 1) : 103-17.

2. Tsou AY, Paulsen EK, Lagedrost SJ, et al. Mortality in Friedreich ataxia. J Neurol Sci 2011 ; 307 : 46-9.
3. Campuzano V, Montermini L, Molto MD, et al. Friedreich's ataxia: autosomal recessive disease caused by an intronic GAA triplet repeat expansion. Science 1996 ; 271 : 1423-7.

4. Perdomini M, Hick A, Puccio H, Pook MA. Animal and cellular models of Friedreich ataxia. J Neurochem $2013 ; 126$ (suppl 1) : 65-79.

5. Perlman SL. A review of Friedreich ataxia clinical trial results. J Child Neurol 2012 ; 27 : 1217-22.

6. Wu Z, Asokan A, Samulski RJ. Adeno-associated virus serotypes: vector toolkit for human gene therapy. Mol Ther 2006 ; 14 : 316-27.

7. Perdomini M, Belbellaa B, Monassier L, et al. Prevention and reversal of severe mitochondrial cardiomyopathy by gene therapy in a mouse model of Friedreich's ataxia. Nat Med $2014 ; 20$ : 542-7.

8. Puccio H, Simon D, Cossee M, et al. Mouse models for Friedreich ataxia exhibit cardiomyopathy, sensory nerve defect and Fe-S enzyme deficiency followed by intramitochondrial iron deposits. Nat Genet 2001 ; $27: 181-6$,

9. Gottlieb RA, Gustafsson AB. Mitochondrial turnover in the heart. Biochim Biophys Acta 2011 ; 1813 : 1295-301.

NOUVELLE

Production de rétines in
vitro à partir de cellules
pluripotentes humaines
Un nouvel outil thérapeutique
Sacha Reichman, José-Alain Sahel, Olivier Goureau

> La rétine, tissu sensoriel qui tapisse le fond de l'œil, est composée d'une monocouche de cellules épithéliales (épithélium pigmentaire rétinien, EPR) et de la neurorétine, qui comprend six types majeurs de neurones organisés de façon stratifiée. Cette dernière permet la conversion du signal lumineux en signal électrique par les photorécepteurs, et le message est transmis jusqu'aux cellules ganglionnaires dont les axones se regroupent pour former le nerf optique qui achemine l'information vers le cerveau. La cécité consécutive aux maladies dégénératives de la rétine, telles que les rétinopathies diabétiques ou héréditaires, la dégénérescence maculaire liée à l'âge (DMLA) et le glaucome, se caractérise principalement par une perte progressive des photorécepteurs, de l'EPR ou des cellules ganglionnaires. Si les techniques de diagnostic ont permis d'améliorer considérablement la classification de ces maladies, l'arsenal thérapeutique est toujours très insuffisant. Des approches alternatives aux traitements pharmacologiques classiques voient le jour telles que les thérapies génique, cellulaire ou prothétique, et restaurer une fonction visuelle perdue apparaît comme un des défis majeurs de la médecine actuelle. Dans ce contexte, la thérapie cellulaire constitue une des approches les plus prometteuses [1]. Les cellules souches
Institut de la vision, Inserm U968; Sorbonne Universités, UPMC-Paris 6, UMR_S968 ; CNRS, UMR 7210, 75012 Paris, France. sacha.reichman@inserm.fr j-sahel@quinze-vingts.fr olivier.goureau@inserm.fr

pluripotentes humaines, telles que les cellules souches embryonnaires ou les cellules pluripotentes induites (cellules iPS, induced pluripotent stem cells), capables de proliférer indéfiniment en culture tout en gardant leur capacité de différenciation, représentent une ressource potentiellement illimitée de cellules rétiniennes pour la transplantation [8]. Un ensemble de travaux récents rapportent la production de photorécepteurs et des cellules de l'EPR à partir de cellules iPS humaines [2, 3]. Cependant, ces méthodes apparaissent peu compatibles avec une large production de cellules d'intérêt thérapeutique. Leur faible efficacité, leur complexité, l'utilisation de composés 